\begin{tabular}{|c|c|c|}
\hline $\begin{array}{l}\text { PKS } \\
\text { PUBLIC } \\
\text { KNOOLEDGE } \\
\text { PROJECT }\end{array}$ & $\begin{array}{c}\text { REVISTA DE GEOGRAFIA } \\
\text { (RECIFE) } \\
\text { http://www.revista.uffe.br/revistageografia }\end{array}$ & $\begin{array}{l}\text { OJS } \\
\frac{\text { OPEN }}{\text { JOUNAL }} \\
\text { SYSTEMS }\end{array}$ \\
\hline
\end{tabular}

\title{
APLICAÇÃO DA SARNDBOX NO ENSINO DE GEOMORFOLOGIA
}

\author{
Riclaudio Silva Santos ${ }^{1}$, Ricardo Silva Santos ${ }^{2}$, Gabriel Carvalho Santos ${ }^{3}$; Kaio César de \\ Oliveira Tavares ${ }^{4}$, Larissa Monteiro Rafael ${ }^{5}$, Daniel Rodrigues de Lira ${ }^{6}$, Cristiano Aprígio \\ dos Santos $^{7}$
}

${ }^{1}$ Universidade Federal de Sergipe - UFS, Email: riclaudio.silva@hotmail.com

${ }^{2}$ Universidade Tiradentes - UNIT, Email: somricardo@hotmail.com

${ }^{3}$ Universidade Federal de Sergipe - UFS, Email: gabrielcontatoufs@gmail.com

${ }^{4}$ Universidade Federal de Pernambuco - UFPE, Email: kaiocesaroliveira@hotmail.com

${ }^{5}$ Universidade Federal de Sergipe - UFS, Email: larissa.rafael@ufs.br

${ }^{6}$ Universidade Federal de Sergipe -UFS, Email: dniellira@ufs.br

${ }^{7}$ Universidade Federal de Sergipe -UFS,Email: aprigeo@gmail.com

Artigo recebido em 10/12/2017 e aceito em 09/03/2018

\begin{abstract}
RESUMO
A Caixa de Areia de Realidade Aumentada (SARndbox) aborda uma metodologia relevante para o ensino de Geociências, em especial a Geomorfologia. Sua utilização, porém, ainda é pouco difundida no Brasil. Essa ferramenta permite a criação de uma representação hipsométrica 3D pelo usuário e, a partir de um senso de movimento, um projetor digital e um conjunto de softwares, modelos topográficos diversos podem ser projetados. Com o objetivo de expor os resultados obtidos com a aplicação da mesma, são apresentados os passos de elaboração da ferramenta, juntamente com os materiais necessários para a sua construção. A SARNDBOX foi aplicada na Universidade Federal de Sergipe, tendo como público-alvo professores e alunos da educação básica, assim como alunos do ensino superior. Com a ferramenta foi possível discutir conceitos da Geomorfologia, assim como demonstrar em modelos topográficos em 3D as características da geomorfologia sergipana.
\end{abstract}

Palavras-chave: Ensino; SARNDBOX; Geomorfologia.

\section{SARNDBOX APPLIANCE FOR GEOMORPHOLOGY TEACHING}

\begin{abstract}
The Augmented Reality Sandbox (SARndbox) represents a relevant teaching methodology for Geosciences, especially Geomorphology. Nevetheless, in Brasil, it has been little applied. This tool allows, by the user, the designing of a 3D hypsometric representation and, from a motion sensor, a digital projector and a set of software, several topographic model can be projected. With the aim to present the methodology application results, this article exposes the tool elaboration steps along with the support material required to build it. SARndbox was applied at the Federal University of Sergipe, targeting Primary and Secondary school teachers and students, as well as Higher Education Students. Thereafter this tool allowed to discuss Geomorphology concept as well as to demonstrate Sergipe's geomorphology characteristics with 3D topographic models.
\end{abstract}

Keywords: Teaching; SARndbox; Geomorphology 


\section{INTRODUÇÃO}

Este projeto, desenvolvido inicialmente em parceria pela Universidade da Califórnia, Centro de Pesquisa Ambiental de Tahoe, e Aquário e Centro de Ciências ECHO Lake. Tratase de uma caixa com areia (sandbox) que, aliada a um sensor de movimento, um projetor digital e um software, geram interações por meio de Realidade Aumentada para a promoção de estudos topográficos, permitindo que usuários criem modelos topográficos em uma superfície e, em tempo real, gera-se uma representação 3D hipsométrica, com linhas de contorno topográficas e água simulada, é possível ainda gerar simulações de erupção vulcânica, inundações, etc. Adicionalmente, são fornecidas sugestões de roteiros de aplicação como apoio ao ensino, pesquisas e uso de conceitos como relevo, legislações (Código Florestal), declividade, entre outros.

A SARNDBOX apresenta-se enquanto uma metodologia inovadora, com reduzidas aplicações no território brasileiro, e com algumas experiências internacionais que demonstram grande potencial metodológico para o ensino das ciências da terra (geociências), e áreas interdisciplinares que trabalham com conceitos da Geologia, Geomorfologia, Pedologia, entre outras. O desenvolvimento desta ferramenta didática tem como objetivo proporcionar um melhor entendimento de processos relacionados a dinâmica do relevo, permitindo a representação em terceira dimensão (3D).

Conforme o propósito do software utilizado na solução, que é o ensino e a visualização de conceitos geográficos, geológicos, geomorfológicos, pedológicos e hidrológicos, o público-alvo deste software é bastante vasto, incluindo: exposições itinerantes de ciência; estudantes do Ensino Fundamental, Médio e Superior; departamentos universitários de ciências da terra, engenharias, entre outros.

O uso das Interfaces Tangíveis é bastante convidativo na área da educação. Muitos estudantes de ensino superior, médio e fundamental apresentam experiências positivas ao utilizar equipamentos práticos e dinâmicos, isso induz a dizer que o equipamento poderia ser utilizado em sala de aula, para tornar uma aula mais didática e atrativa.

A maior contribuição brasileira para a elaboração do projeto foi o Manual de instalação, configuração e uso da caixa de areia de realidade aumentada (SARndbox) (KAWAMOTO, 2016). Na Universidade Federal de Sergipe, o projeto da SARNDBOX foi desenvolvido pelo Grupo de Pesquisa em Geomorfologia do Quaternário e Modelagem Ambiental - QUACOMA, e aplicada durante a IV SEMAC - Semana Acadêmico-cultural da 
UFS, na ação de extensão Modelagem Ambiental em Realidade Aumentada: Aplicação da Caixa de Areia, e posteriormente será utilizada para as demais atividades acadêmicas, como disciplinas, projetos, oficinas, minicursos, exposições e etc.

\section{MATERIAIS E MÉTODOS}

A construção da SARndbox seguiu o manual elaborado por Kawamoto (2016), com algumas adaptações. Como também os trabalhos desenvolvidos pela International Organization for Standardization, Augmented Reality Sandbox, e outras referências encontradas em fóruns internacionais que discutem a aplicação desta ferramenta, que forneceram informações para instalação e configuração dos softwares para o funcionamento do projeto.

A SARndbox ou Caixa de Areia de Realidade Aumentada, requer para seu funcionamento, os seguintes equipamentos: Um computador com placa gráfica dedicada (offboard), executando Sistema Operacional Linux; Um sensor de profundidade Microsoft Kinect 1.0; O software utilizado pela ARS (Kinect 3D Video Package) é compatível com os três modelos da primeira geração Kinect (Kinect para a Xbox 1414, 1473 e Kinect para Windows); Um projetor digital de dados com uma interface de vídeo digital, como HDMI, DVI ou DisplayPort; Uma caixa de areia de forma que seja possível instalar o sensor Kinect e o projetor acima.

Para executar o software da ARS, recomenda-se utilizar um computador com placa de vídeo offboard como, por exemplo, uma AMD/ATI Radeon ou NVidia GeForce. O processador (CPU) do computador deve ser rápido (Intel Core i5 é suficiente). O sistema não requer grande quantidade de memória RAM, sendo que 2 GB são suficientes. Quanto ao disco rígido (Hard Disk - HD), 20 GB bastam para a instalação do Sistema Operacional e do software. Embora existam relatos de execução desse sistema em Sistemas Operacionais MacOS X, os desenvolvedores recomendam utilizar Linux por questões de estabilidade na execução.

O Sistema Operacional Linux Mint, versão MATE de 64-bit. O sistema da ARS possui dois componentes principais: um renderizador do mapa topográfico - que gera as curvas projetadas sobre a superfície da areia, e um componente responsável pela simulação de fluxo de água. O primeiro componente executa com relativa facilidade em processador e placa de vídeo de desempenho médio (mid-end) e funciona na maioria dos laptops ou PCs disponíveis no mercado. 
O componente de simulação de água, por sua vez, requer hardware de um alto desempenho (high-end), razão da recomendação de utilizar uma placa de vídeo como a Nvidia GeForce GTX 970, ou superior, capaz de processar dados gráficos e gerar animações em tempo real. Idealmente, o projetor deve ter um comprimento de curta distância e uma proporção dimensional nativa de 4:3 para coincidir com o campo de visão da câmera do sensor Kinect.

A resolução de $1024 \times 768$ pixels é suficiente, uma vez que a resolução total do sistema é limitada pela câmera do Kinect (640x480 pixels). O projetor ideal é do tipo "projetor de linha central", para ser montado diretamente ao lado da câmera Kinect. Finalmente, não é recomendado utilizar conexão analógica (VGA) entre o projetor e o computador, pois a qualidade de imagem pode ser degradada, além de introduzir desalinhamento entre a imagem projetada e a superfície de areia.

A caixa utilizada deve ter uma proporção de 4:3, para corresponder tanto ao campo de visão da câmera do Kinect quanto à área de cobertura do projetor. O tamanho da caixa é limitado pelas distâncias mínimas e máximas da câmera Kinect e pela resolução desejada. O ângulo do campo de visão da câmera do sensor Kinect é de cerca de $90^{\circ}$, por isso, o sensor deve ser posicionado o mais alto possível acima da superfície da areia, diretamente acima do centro da caixa.

A caixa de areia deve ser preenchida com areia a uma profundidade de cerca de $4 \mathrm{~cm}$ a $10 \mathrm{~cm}$. Os desenvolvedores recomendam o uso do produto comercial Sandtastik White Play Sand, que possui excelentes propriedades de projeção. É recomendado manter a areia ligeiramente úmida para facilitar a criação de contornos.

\section{RESULTADOS E DISCUSSÕES}

Com a construção e aplicação da caixa de areia de realidade aumentada foi possível discutir e trabalhar várias questões envolvendo a geomorfologia e demais geociências. Além da discussão envolvendo os conteúdos, os próprios alunos puderam interagir com a caixa de areia para criar formas de relevo (Fig. 01 e Fig. 02). Na Fig 01, Alunos da rede estadual de ensino fazendo uso da caixa de areia em realidade aumentada, Na Fig 02, Alunos da Universidade Federal de Sergipe fazendo uso da caixa de areia em realidade aumentada. 
Revista de Geografia (Recife) V. 35, No. 4 (especial XII SINAGEO), 2018

Figura 01 - Alunos do ensino médio utilizando a SARndbox.
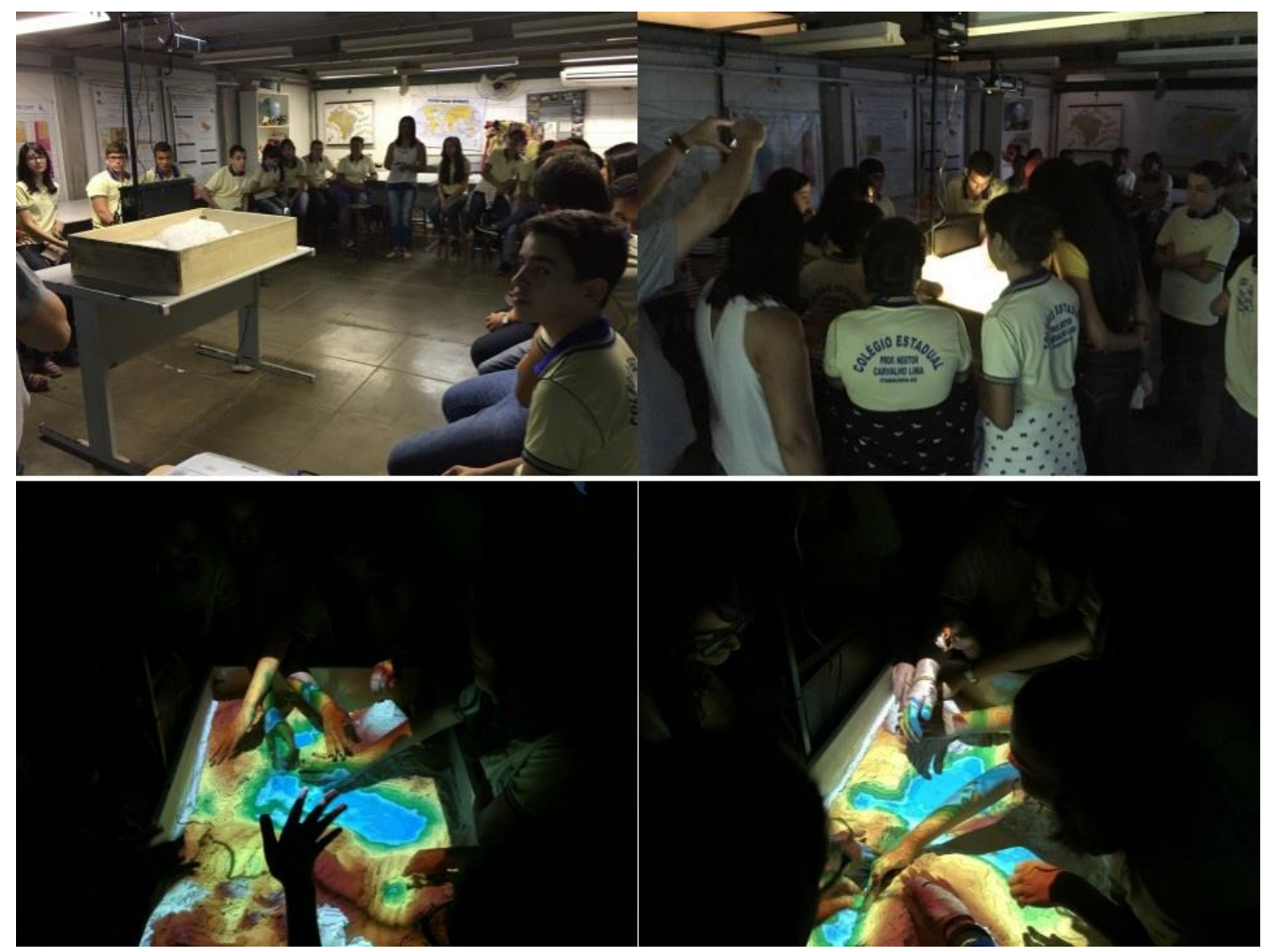

Figura 02 - Alunos do ensino superior utilizando a SARndbox.
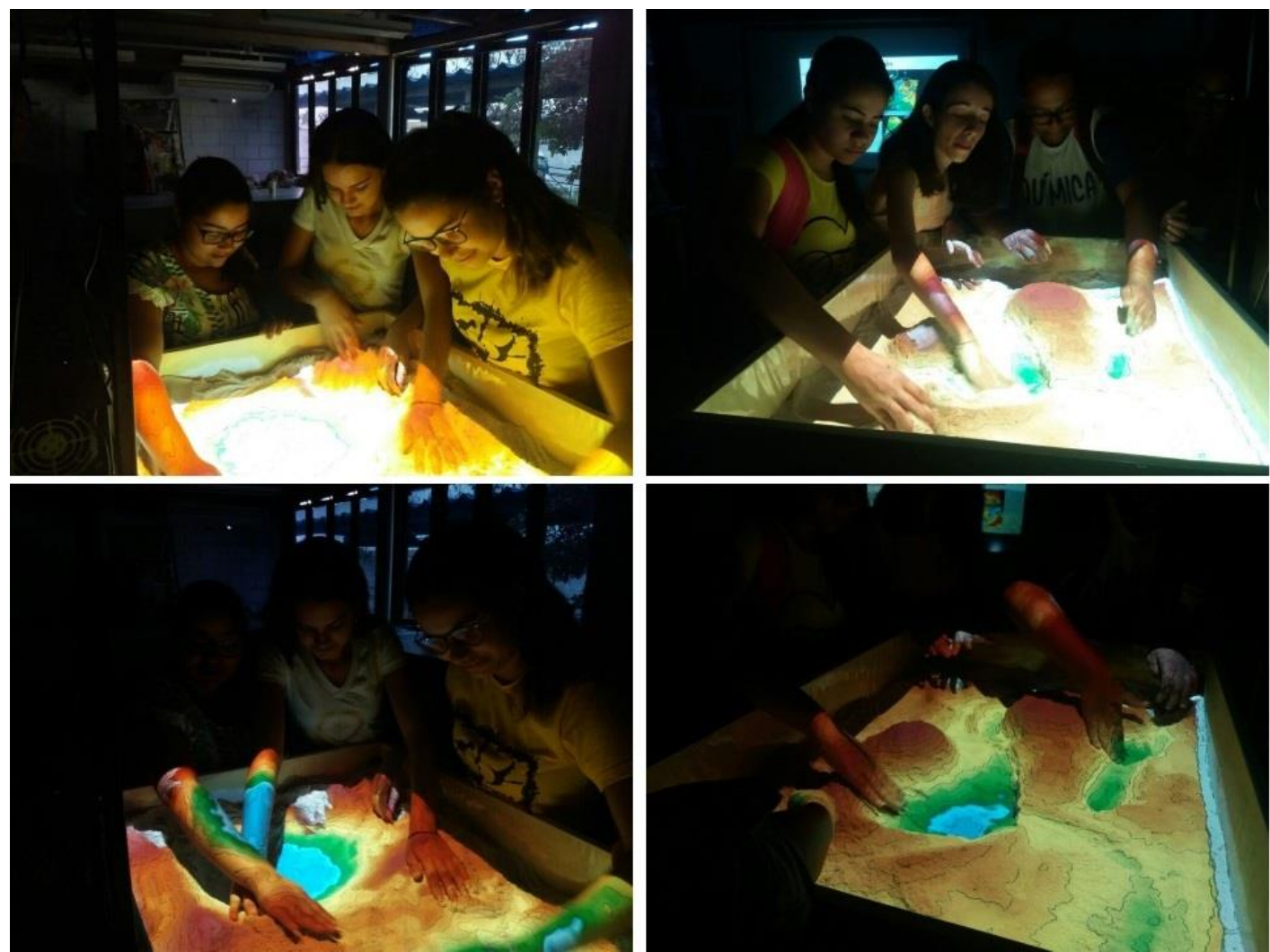
Entre as aplicações que foram desenvolvidas com esta ferramenta, podemos destacar as seguintes. Foram construídas na areia, diferentes formas de relevo, trabalhando conceitos relacionados com cada forma, sua gênese e evolução. As formas de relevo construídas foram morros, colinas, chapadas, planícies, entre outras (Fig. 03)

Figura 03 - Formas de relevo criadas pelos participantes da atividade.
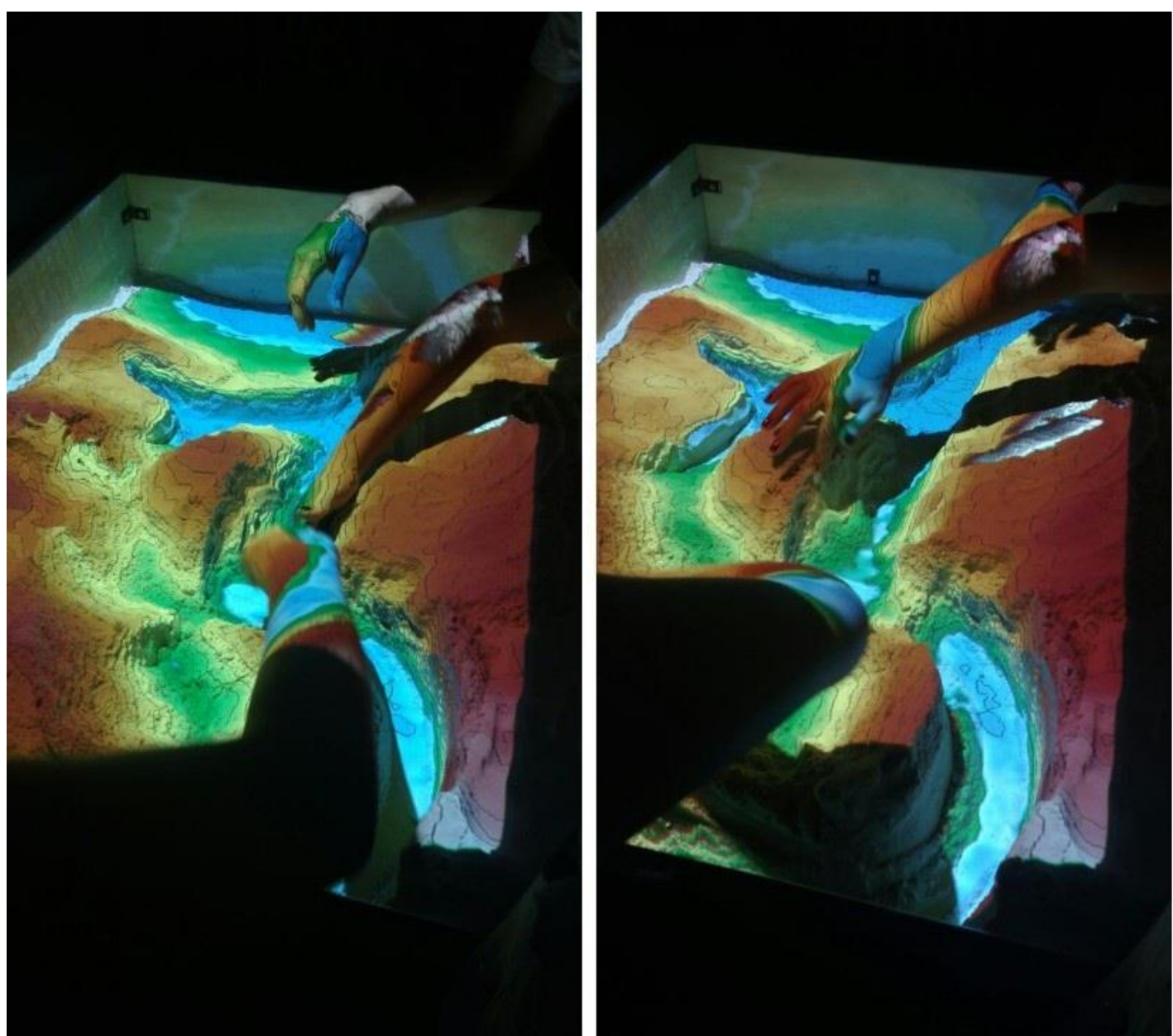

Fonte: Os autores (2017).

Acima formas representativas de relevo criadas pelos próprios participantes da atividade, para a discussão de conceitos geomorfológicos (fig 04). Foi trabalhada a relação da rede de drenagem, dos rios, da bacia hidrográfica com o relevo e com o contexto de uso e ocupação, envolvendo temas como poluição, contaminação, enchentes, mata ciliar, entre outros. Com a criação de formas de relevo, calculamos a declividade e classificamos as formas construídas conforme a relação proposta pela Embrapa, em relevo plano (0- 3\%), suave ondulado (3-8\%), ondulado (8-20\%), forte ondulado (20-45\%), motanhoso (45-75\%), escarpado $(>75 \%)$. 
Figura 04 - Representaçôes do relevo na SARndbox.
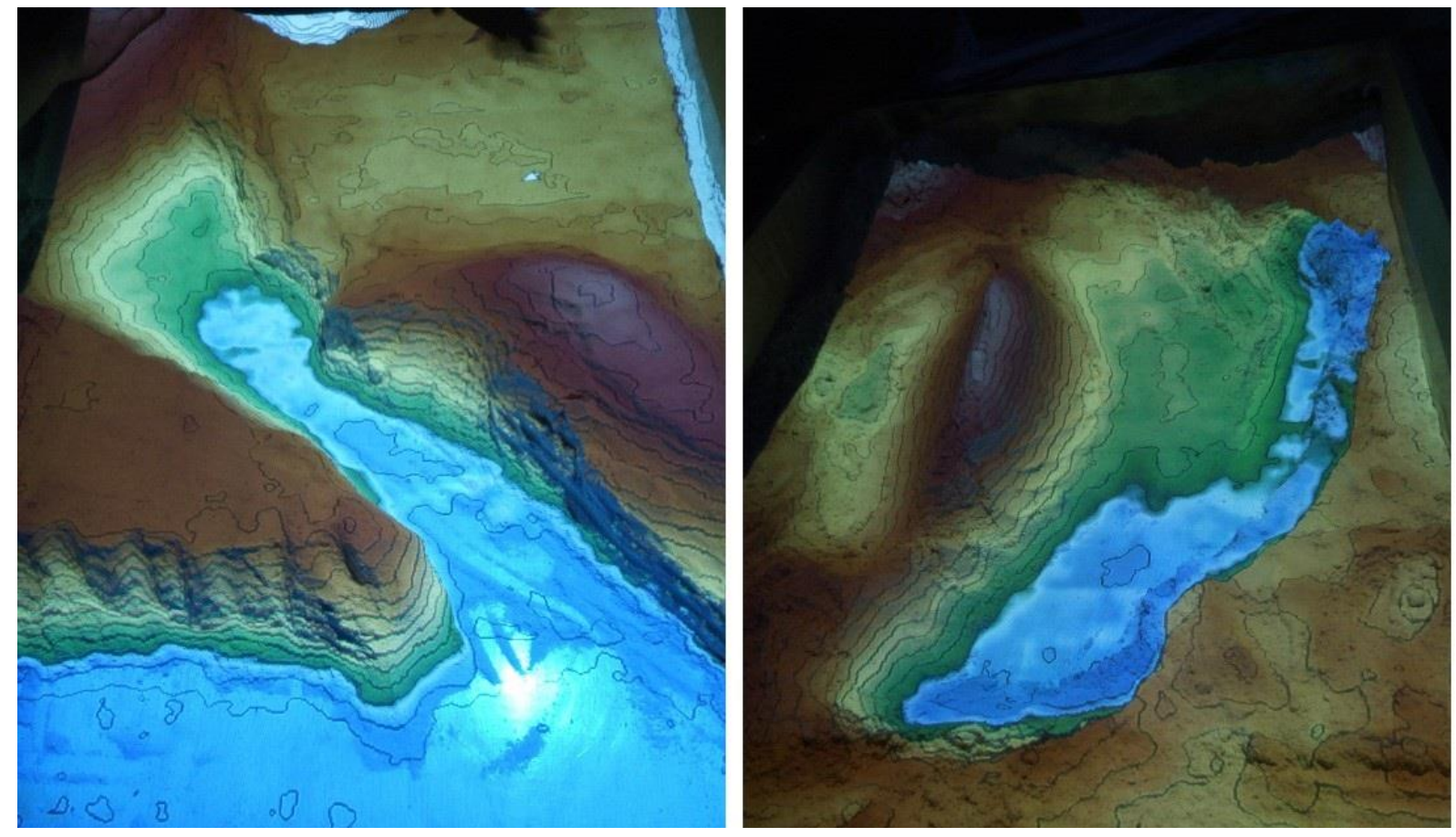

Observamos na figura 4, representações do relevo utilizadas para o debate de conceitos geomorfológicos e das demais geociências. Reproduzimos formas de relevo com declividades que estariam dentro dos limites de uso conforme o Novo Código Florestal (Lei $\mathrm{N}^{\mathrm{o}}$ 12.651/2012) estabelece. Esta aplicação envolve temas como vertentes/encostas, uso e ocupação da terra, relação meio ambiente com leis ambientais, deslizamentos, cortes e aterros, entre outros. Essas e outras utilizações da SARndbox também podem ser encontradas no manual da caixa de areia (KAWAMOTO, 2016).

Além dessas aplicações foi possível trabalhar os diferentes contextos geomorfológicos encontrados no estado de Sergipe, partindo da depressão sertaneja, onde foram representados os maciços cristalinos, as áreas de pedimento, vale e planície fluvial, os inselbergs (Monadnocks). O Domo de Itabaiana, onde foram demonstradas as serras que compõem as bordas do domo, as rampas de colúvio, áreas de pedimento, a relação entre relevo e estrutura geológica que é tão marcante na região. E também representações da planície costeira, discutindo processos erosivos, de variação da linha de costa, e as fragilidades encontradas em ambientes flúvio- marinhos.

A aplicação da SARndbox atingiu um público de 237 pessoas inscritas no evento, contando com a presença de alunos do ensino fundamental e médio da rede de ensino estadual e também de alunos do curso de Licenciatura em Geografia, Biologia, Matemática, Física, 
Química e Letras, assim como da Engenharia Civil e de Sistemas de Informação. Quando questionados, os participantes do evento demonstraram satisfação em participar da dinâmica envolvendo o uso da caixa de areia, relatando ainda que, deste modo, os assuntos trabalhados em sala de aula tornam-se muito mais fáceis de serem compreendidos.

O impacto positivo pôde ser notado tanto para os alunos de nível superior quanto para os do ensino fundamental, que demonstravam um grande interesse em participar das atividades, questionando a possibilidade de terem o equipamento didático em sua escola para as aulas de Geografia.

\section{CONSIDERAÇÕES FINAIS}

A aplicação da caixa de areia em realidade aumentada mostrou bons resultados enquanto uma metodologia de ensino para a geomorfologia, como também para as demais geociências. A possibilidade de utilizar uma ferramenta interativa, onde o próprio aluno pode construir as formas de relevo, e visualiza-las em 3D contribui de maneira ímpar para o ensino dos temas que envolvem a geomorfologia, tanto num caráter próprio desta ciência como em estudos interdisciplinares podendo correlaciona-los também com a geologia, pedologia, topografia, e demais ciências da terra.

Novas tecnologias estão cada vez mais presentes no cotidiano, ferramentas com interface tangíveis estão cada vez mais deixando de ser um conceito e se tornando realidade. O Augmented Reality Sandbox é um exemplo de um equipamento com diversas aplicações na docência. Sua utilização simples e agradável, como mostra este artigo, pode trazer mais atenção a esta tecnologia que pode se tornar um dia, parte do nosso cotidiano estudantil.

\section{REFERÊNCIAS}

Augmented Reality Sandbox - Disponível em: http://idav.ucdavis.edu/ okreylos/index.html Acesso em: 14 nov. 2016.

International Organization for Standardization. Ergonomics of human system interaction, 2009, Part 210: Human-centered design for interactive systems (formerly known as 13407). ISO 9241-210:2009, Disponível em: <https://www.iso.org/obp/ui/\#iso:std:iso:9241:-210:ed1:v1:en> Acesso em 13 nov. 2016.

KAWAMOTO, André Luiz Satoshi. Manual de instalação, configuração e uso da caixa de areia de realidade aumentada (SARndbox). Manual - Universidade Tecnológica Federal do 
Paraná, Departamento de Ciências da Computação e Departamento Acadêmico de Ambiental, Campo Mourão-PR. 2016.

SARndbox UTFPR: Projeto de Recursos Educacionais abertos - Elaboração de Material de Apoio Didático Utilizando Dispositivo de Baixo Custo para Interfaces Naturais. Apoio: UTFPR-CM; DIRGRAD; DIREC; DIRPPG, disponível em: https://github.com/projetosar/SARndbox/blob/master/manualSarndboxUTFPRCM.pdf. Acesso em 14 nov. 2016. 